\title{
AIAA 97-0750
}

Large-Eddy Simulation of Laminar-Turbulent Transition in a Swept-Wing Boundary Layer

$X$. Huai

University of Maryland

College Park, MD

R. D. Joslin

NASA Langley Research Center

Hampton, VA; and

U. Piomelli

University of Maryland

College Park, MD 


\title{
LARGE-EDDY SIMULATION OF LAMINAR-TURBULENT TRANSITION IN A SWEPT-WING BOUNDARY LAYER
}

\author{
Xiaoli Huai* \\ University of Maryland \\ College Park, MD 20742 \\ Ronald D. Joslin ${ }^{\dagger}$ \\ NASA Langley Research Center \\ Hampton, VA 23681 \\ and \\ Ugo Piomelli \\ University of Maryland \\ College Park, MD 20742
}

\begin{abstract}
$\underline{\text { Abstract }}$
The spatial development of laminar-turbulent transition in a $45^{\circ}$ swept-wing boundary layer is investigated using the large-eddy simulation approach. Both stationary and traveling disturbances are initiated by steady and random forcings. The numerical simulations reproduce the surface shear streaks typical of the crossflow instability observed in experiments. Downstream of the transition location the wall shear stress is found to turn towards the streak direction. The strength of the stationary vortices grows exponentially, initially independent of the unsteady forcing amplitude and saturates at different levels during the later development, depending on the magnitude of the traveling vortices. Streamwise velocity contours show the evolution from a wave-like structure to the "half-mushroom" structure for the lower-amplitude, traveling-wave case, leading to double inflection points in the wallnormal velocity profiles prior to transition; the nonlinear interactions involving the stationary vortices are also much stronger, as indicated by the amplification of higher harmonics of the primary mode. The frequency spectrum of the traveling waves show a high frequency secondary instability prior to transition that, however, is not prominent in the higheramplitude traveling-wave case.
\end{abstract}

\footnotetext{
* Current address: AEA Technology Engineering Software Inc., 2000 Oxford Drive, Pittsburgh, PA 15102

${ }_{\dagger}$ Leader, Laminar Flow Control Project Team, Member AIAA.

$\ddagger$ Associate Professor, Department of Mechanical Engineering. Senior Member AIAA.
}

\section{$\underline{\text { Introduction }}$}

The transition process in a swept-wing boundary layer is significantly different and more complex than transition on a flat plate. One important type of instability that exists in the swept-wing boundary layer is the so-called crossflow instability, which is caused by the inflection point present in the secondary (crossflow) velocity profile within the boundary layer. This instability plays a dominant role in the favorable pressure gradient region where the Tollmien-Schlichting waves are suppressed.

The most distinctive feature of crossflow instability is the existence of co-rotating stationary and traveling vortices, also known as crossflow vortices. The stationary vortices, which are initiated by surface roughness, tend to be aligned approximately in the streamline direction, and were observed in flowvisualization experiments (Dagenhart, 1992). Prior to transition, the velocity field is so strongly influenced by the stationary vortices that the streamwise velocity profiles may exhibit multiple inflection points (Dagenhart, 1992; Joslin and Streett, 1994; Joslin, 1995). These inflectional profiles generate higher-frequency, secondary-instability traveling modes (Kohama et al., 1992). Experiments also showed that traveling vortices, especially the secondary instability modes, play an important role in driving the laminar flow to transition and turbulence (Müller and Bippes, 1988).

Despite many theoretical and experimental studies, the current understanding of transition in a swept-wing boundary layer is still incomplete because of the complexity of the problem, and because of the difficulties in carefully controlling the experimental conditions and the inclusion of nonlinear 
effects in theoretical studies. Numerical simulations employing the full Navier-Stokes equations - direct numerical simulations (DNS) - can, therefore, give a substantial contribution to the understanding of the flow physics because the ingestion of disturbances into the computed boundary-layer can be more carefully controlled than in experiments. The cost of DNS limits, however, its application to flows of engineering interest at high Reynolds number. Even at moderate or low Reynolds numbers, the computational effort required to perform a calculation of the full transition from laminar to turbulent flow is extremely high.

Large-eddy simulation (LES) is an alternative, less expensive, approach for the numerical solution of turbulent and transitional flow problems. Unlike DNS in which all scales of motion are resolved, only the dynamically important large scales need to be resolved in LES, which results in a significant decrease of the CPU time required for a simulation compared with DNS. The influence of the unresolved small scales on the large scales is modeled. Since the small scales tend to be more homogeneous and isotropic than the large scales (which are highly flow-dependent), the modeling can be simpler and more universal than that used in the Reynoldsaveraged approach.

The objective of this work is to study of transition in a swept-wing boundary layer using the largeeddy simulation approach. Two simulations are conducted with small and large initial amplitudes of the time-dependent disturbances and fixed amplitudes of the stationary disturbances. The effect of random, three-dimensional, and time-dependent disturbances on the flow breakdown, and the interaction between the stationary vortices typical of swept-wing transition and the traveling modes generated by the time-dependent disturbance will be investigated to elucidate the mechanisms by which laminar flow becomes turbulent.

\section{Numerical Method}

In the present study, the filtered governing equations for the disturbance variables $\left(u_{i}, p\right)$ are in the form:

$$
\begin{array}{r}
\frac{\partial \bar{u}_{i}}{\partial t}+\bar{u}_{j} \frac{\partial \bar{u}_{i}}{\partial x_{j}}+U_{o j} \frac{\partial \bar{u}_{i}}{\partial x_{j}}+\bar{u}_{j} \frac{\partial U_{o i}}{\partial x_{j}}= \\
-\frac{\partial \bar{p}}{\partial x_{i}}-\frac{\partial \tau_{i j}}{\partial x_{j}}+\frac{1}{R e} \frac{\partial^{2} \bar{u}_{i}}{\partial x_{j} \partial x_{j}}
\end{array}
$$

and

$$
\frac{\partial \bar{u}_{i}}{\partial x_{i}}=0,
$$

where an overbar denotes the application of the filtering operation, and $\tau_{i j}=\overline{u_{i} u_{j}}-\bar{u}_{i} \bar{u}_{j}$ is the subgrid-scale (SGS) stress tensor, which must be modeled. The base flow field $\left(U_{o i}\right)$ is given by the Falkner-Skan-Cooke similarity solution (Cooke, 1950). The Reynolds number is $R e=Q_{r} \delta / \nu$, where $Q_{r}$ is the local edge velocity at the computational inflow boundary, and $\delta=\sqrt{\nu x_{c} / U_{o e}}$ is the reference length at the inflow.

The filtered Navier-Stokes equations are solved using the fractional time step method (Chorin, 1968). Fourth-order finite difference and fourthorder compact difference schemes are used in the streamwise direction $(x)$ for the pressure and the momentum equations, respectively, Chebychev series are used in the wall-normal direction $(y)$, and Fourier series in the spanwise direction $(z)$. The implicit Crank-Nicolson time-advancement is used for the wall-normal diffusion terms and a threestage Runge-Kutta scheme for the remaining terms (Joslin et al., 1992). The subgrid-scale stress term is modeled by a localized version (Piomelli and Liu, 1995) of the dynamic SGS model (Germano et al., 1991). Spanwise averaging as well as three point averaging in the streamwise and wall-normal directions are used when calculating the localized dynamic SGS model coefficient. The total viscosity (molecular + eddy viscosity) is also forced to be non-negative to ensure numerical stability.

One of the major difficulties associated with the numerical simulation of spatially developing boundary-layer transition is to define the outflow boundary conditions. Here the buffer domain technique proposed by Streett and Macaraeg (1989) is used, in which the governing equations are gradually parabolized in a buffer region which is appended at the end of the computational domain, thus eliminating the necessity of applying the outflow boundary conditions. A fourth-order compact-difference filter (Lele, 1992) is used in the streamwise direction every 10 time steps, to eliminate numerical wave reflections at the outflow (Pruett et al., 1995).

\section{$\underline{\text { Simulation Parameters }}$}

In the present simulations, some of the parameters were chosen to match the DFVLR $45^{\circ}$ swept flat-plate experiment carried out by Müller and Bippes (1988). The base flow was determined by 
imposing the sweep angle and the pressure distribution

$$
c_{p}\left(x_{c}\right)=0.941-0.845 x_{c}
$$

on the Falkner-Skan-Cooke equations. Two computational boxes with different grid resolutions were used to cover the whole physical domain to be studied, and the inflow conditions for the second box were read from the outflow of the first one. This implementation was used successfully in the largeeddy simulation of subharmonic breakdown in a flatplate boundary layer (Huai, 1996; Huai et al., 1997). Near the computational inflow, small disturbances were introduced by imposing a wall-normal velocity distribution in the form of a suction and blowing strip. A dominant stationary vortex mode was explicitly introduced by applying steady suction and blowing. The dominant spanwise wavelength was found to be $\lambda_{z}=36$ for the conditions used in Joslin and Streett (1994), and the same conditions and dominant wavelength were used in the present study. Traveling crossflow vortices were introduced into the flow by random modulations of the suction and blowing at each time step. The zero-frequency mode was filtered out from the random part of the suction and blowing to prevent the traveling-wave generating mechanism from altering the initial stationary wave, and also to maintain overall mass conservation. Since no other restriction was applied, the random function contained all the non-zero frequency and wavenumber modes allowed by the grid resolution, time step size and time interval of the data sampling.

Two simulations, denoted by LOW-T and HIGH$\mathrm{T}$, were carried out. While the steady suction and blowing had the same magnitude in both simulations $\left(A_{o}=10^{-4}\right.$, where $A_{o}$ is the amplitude of the wall-normal velocity fluctuation in the suction strip), the magnitudes of the unsteady suction and blowing, $A_{t}$, were different, with $A_{t}=10^{-4}$ and $10^{-2}$ for the LOW-T the HIGH-T cases, respectively, to study the role of traveling vortices and their effects on the transition process. The Reynolds number at the inflow was 998 , which is equivalent to a chord Reynolds number $R e_{c}=2.73 \times 10^{6}$. The two simulations covered the chordwise regions of $0.2<x_{c}<0.54$ and $0.2<x_{c}<0.5$, and required about 100 and 90 Cray C-90 hours respectively. The two boxes for LOW-T had streamwise $\times$ wallnormal $\times$ spanwise lengths of $358 \times 50 \times 108$ and $386 \times 50 \times 108$, and used $326 \times 49 \times 33$ and $461 \times 61 \times 49$ grid points. The two boxes for HIGH-T had do- main lengths of $272 \times 50 \times 108$ and $386 \times 50 \times 108$, and used $266 \times 49 \times 33$ and $461 \times 61 \times 49$ grid points respectively.

\section{Results}

\section{Code validation}

The numerical scheme and SGS models had previously been validated in two studies: the first was the calculation of subharmonic instability in a flat-plate boundary layer (Huai, 1996; Huai et al., 1997); the second, the calculation of transition in a swept wing in presence of stationary disturbances only (Huai et al., 1994).

The first calculation showed good agreement between the LES results and linear theory in the early transition stages, and excellent agreement with the data obtained from DNS calculations (Spalart, 1986). The second simulation studied a flow very similar to the one under consideration here; the results were compared with the DNS data of Joslin and Streett (1994), and good agreement was achieved between the LES and DNS data.

A thorough grid-resolution study could not be performed due to the computational expense involved; however, one test was run using the same conditions as case HIGH-T, but a refined mesh in the second box (three times as many points in $x$, and twice as many in $z$; the spanwise domain was onethird of that used in simulation HIGH-T, however). The fine-mesh calculation was in good qualitative agreement with the coarser one; for $x_{c}<0.42$, the agreement was also quantitatively satisfactory.

A direct comparison of the present LES results with experimental or theoretical data was not performed in this study, given the wide variety of flow conditions used in the previous studies. Several flow features obtained from the LES agree well with experimental and theoretical data (see below). Based on the detailed comparisons of the LES results with DNS for flat-plate boundary-layer transition and the grid refinement comparisons for the present threedimensional flow reported by Huai (1996), it is possible to conclude that the early nonlinear stages of transition are captured quite accurately, while the marginal grid resolution in the final breakdown stages allows us to draw only qualitative conclusions regarding the transition itself.

\section{Flow visualization}

In flow visualization experiments of threedimensional boundary-layer transition, surface 

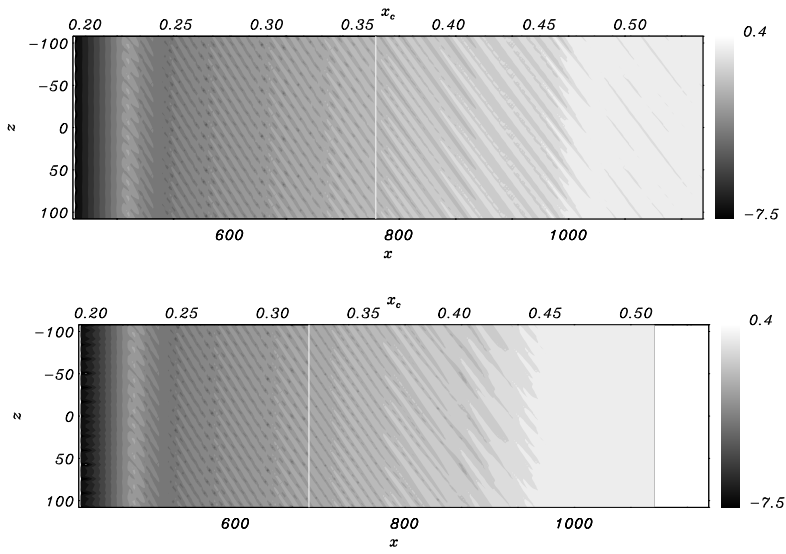

Figure 1. Contours of mean wall shear $\log _{10}(\partial V / \partial y)_{w}$. Top: LOW-T; bottom: HIGH-T. The white vertical lines represent the junction between the first and the second boxes.

streaks, which are the signatures of stationary crossflow vortices, have been frequently observed. Poll (1985) was able to observe the surface streaks in a swept cylinder boundary layer. Dagenhart (1992) documented the stationary crossflow vortices in a swept-wing boundary layer by taking photographs of the naphthalene flow visualization: a white naphthalene sublimating coating was placed over the black swept-wing surface; the existence of stationary crossflow vortices gave rise to alternating highand low-shear regions on the wall.

Contours of mean wall shear from the present simulations are shown in Fig. 1 (two computational domains are shown side by side in each figure). The alternating high- and low-shear regions are evident. As in the experiment of Dagenhart (1992), no vortex merging or adjustment of vortex spacing is observed, in contrast to the experimental results of Arnal and Juillen (1987). The vortex adjustment in the numerical simulations could be restricted to some extent by the spanwise periodicity.

The transition location can be estimated in Fig. 1 as the place where the distinct streak patterns become blurred or disappear. For LOW-T and HIGH$\mathrm{T}$ they are at $x_{c} \simeq 0.45$ and 0.42 respectively. Transition occurs earlier in the higher time-dependent disturbance (HIGH-T) case. This result is consistent with the experimental results of Müller and Bippes (1988), who suggested that the traveling waves play the most important role in determining the onset of transition, while large amplitudes of the stationary vortices do not seem to advance transition. Another interesting observation is that in the
LOW-T case the change of the streak patterns in the downstream region is milder and remnants of the stationary vortices extend deep into the transition and turbulent regions.

\section{Development of the mean quantities}

Time- and spanwise-averages were computed from the sampled flow-field data to obtain meanflow quantities. Instead of using the velocity components in the body-oriented coordinate frame $(x, y, z)$, the velocities were projected into the floworiented coordinate system $\left(x_{s}, y_{s}, z_{s}\right)$ defined by the local external streamwise $\left(x_{s}\right)$ and the crossflow $\left(z_{s}\right)$ directions. The displacement thickness $\delta_{s}^{*}$, momentum thickness $\Theta_{s}$, shape factor $H_{s}$ and skinfriction coefficient $C_{f, s}$ associated with the streamwise mean velocity $U_{s}$ are defined in this coordinate system as

$$
\begin{aligned}
\delta_{s}^{*} & =\int_{0}^{y_{\max }}\left(1-\frac{U_{s}}{U_{s, e}}\right) d y, \\
\Theta_{s} & =\int_{0}^{y_{\max }} \frac{U_{s}}{U_{s, e}}\left(1-\frac{U_{s}}{U_{s, e}}\right) d y, \\
H_{s} & =\frac{\delta_{s}^{*}}{\Theta_{s}},
\end{aligned}
$$

and

$$
C_{f, s}=\frac{\tau_{w, s}}{\frac{1}{2} \rho U_{s, e}^{2}},
$$

where $U_{s, e}$ is the boundary-layer edge velocity.

Figure 2 shows the chordwise variation of the skin-friction coefficient and shape factor for the LOW-T and HIGH-T cases. Initially, while the disturbances are growing, the mean flow is unaltered from its laminar state. As the crossflow disturbances amplify, however, the mean flow begins to be affected by the disturbances, the skin friction increases and the shape factor decreases from their laminar values, indicating the onset of transition to turbulence in imminent. Finally the curves level off to a turbulent state. The higher timedependent perturbation case, HIGH-T, results in earlier transition than LOW-T, but the differences between the shape factors are small in the early transition regions and become pronounced only in the later stages of transition. The laminar shape factor is lower than the flat-plate boundary-layer value of 2.6, because of the three-dimensionality of the boundary-layer flow. The turbulent values are almost the same for both LOW-T and HIGH-T, regardless of the difference in $A_{t}$. 

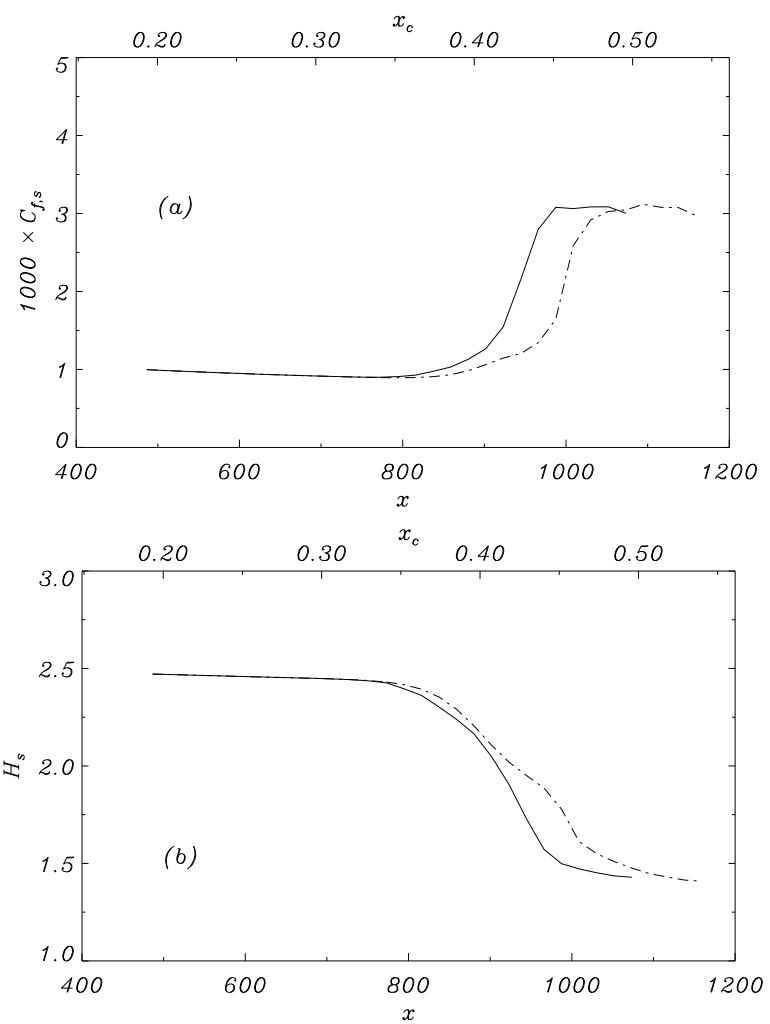

Figure 2. Chordwise variation of the (a): the skin friction coefficient $C_{f, s}$, and (b): shape factor $H_{s}$. -.— : LOW-T; — HIGH-T.

Due to both the sweep angle of the flow and the pressure gradient, the streamlines and wall shearstress change direction with chordwise location. As shown by Joslin and Streett (1994), the crossflow vortices follow the external streamwise direction, within a few degrees. The angles obtained in the present study by measuring the streak patterns are plotted in Fig. 3. The deviation, $\varepsilon=4^{\circ}$, from the direction of the external streamwise direction $\beta$ matches the experimental result of $\varepsilon=$ $3^{\circ} \sim 5^{\circ}$ (Nitschke-Kowsky and Bippes, 1988). The wall shear-stress initially follows the laminar basestate direction; as transition occurs, however, the strength of the crossflow disturbances affects the near-wall flow and the shear direction switches from the laminar state to the direction of the crossflow vortex disturbance.

\section{Development of the stationary vortices}

In Fig. 4, the maximum streamwise velocity associated with the stationary vortices for both simulations is shown. Irrespective of the different

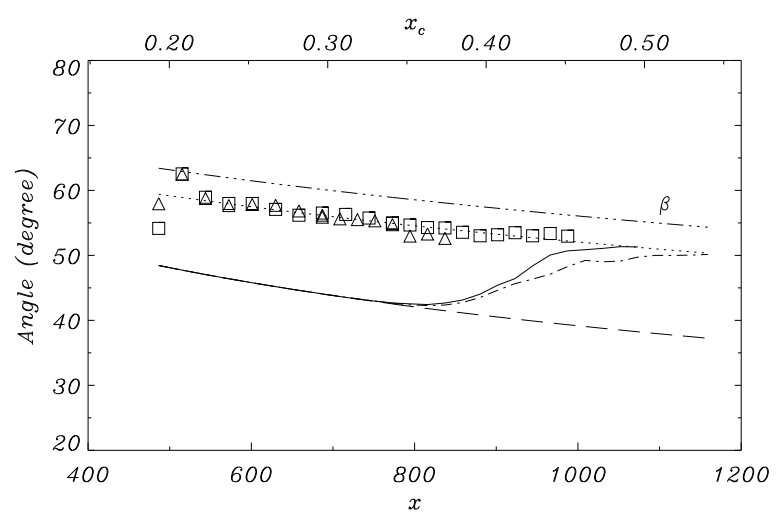

Figure 3. Chordwise variation of velocity, vortex and wall-shear angles. External streamwise angle $\beta$ : $-\cdots-$; stationary crossflow vortex angle $\beta-\epsilon$, LOW-T: $\square$, HIGH-T: $\triangle ; \beta-4^{\circ}$ : $\cdots \cdots$; wall shearstress angle of the laminar base flow: - wall shear-stress angle, LOW-T: - - - HIGH-T:

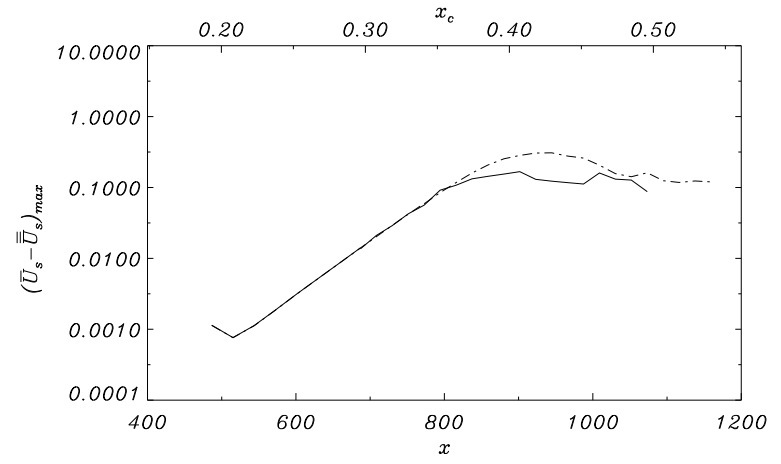

Figure 4. Chordwise growth of the stationary disturbances. - - L LW-T; - HIGH-T.

amplitudes of the traveling vortices, both simulations give stationary disturbances with exponential growth rates $\alpha_{i}=11$. This growth rate is very close to the experimental result, $\alpha_{i}=13 \pm 1.3$, of Müller and Bippes (1988) for the $T u=0.05 \%$ case, given the experimental uncertainty. The experimental growth was also in good agreement with results $\left(\alpha_{i}=12\right)$ of a numerical calculation (Meyer, 1987). However, the experiment also showed that the initial amplification of the stationary vortices was lower for the higher freestream turbulence, i.e., $T u=0.15 \%$, case, apparently in contrast with the present results, that yield the same growth rate for both HIGH-T and LOW-T cases. In the $T u=0.15 \%$ case, however, the initial magnitude of the traveling disturbance was about the same as that of the stationary one, 
and about half that of the stationary disturbance for the low turbulence case. In the present simulations, on the other hand, the traveling disturbance is weaker, its amplitudes is close to the experimental $T u=0.05 \%$ case in HIGH-T, and one order of magnitude smaller than the stationary wave; in LOW-T, the traveling wave is three orders of magnitude smaller than the stationary wave. The present results, combined with the experimental findings, seem to suggest that if the magnitude of the traveling waves has not exceeded a certain threshold (somewhere between 0.5 and 1 of the amplitude of the stationary waves), the initial amplification of the stationary crossflow vortices can be predicted satisfactorily by the theory (Meyer, 1987); otherwise it will be lower than the theoretical prediction. The reason for this is that the accelerated nonlinear interactions transfer energy from the stationary waves to the traveling ones.

The saturation (or over-saturation for LOW-T) of the stationary vortices prior to transition can also be observed in Fig. 4. It starts at different chordwise distances depending on the locations of transition. This observation is in agreement with the findings of the crossflow instability experiment of Müller and Bippes (1988), which showed that earlier transition in the case of stronger traveling waves results in earlier saturation.

Figure 4 shows the growth of mean distortion of the laminar base flow, but it provides no information on the wavenumber content of the stationary vortices, their growth, and on the possible nonlinear wave interactions that may play an important role in understanding the transition process. For example, in the crossflow experiment of Saric and Yeates (1985), the stationary vortices having the wavelength of $1 \mathrm{~cm}$ predicted by linear theory were observed, while in the hot-wire measurements the second harmonic having a wavelength of $0.5 \mathrm{~cm}$ away from the wall was also found, and its characteristics amplitude was as high as three times that of the $1 \mathrm{~cm}$ primary wave. Reed (1987) studied wave interactions in the framework of secondary instability and concluded that the anomalies found in the experiment are introduced by a crossflow/crossflow interaction between the primary vortices and nonstationary disturbances whose wave-length is half that of the primary crossflow vortices.

To investigate the nonlinear interactions that occur in this flow, the spanwise wavenumber spectra of the steady streamwise perturbation velocity were calculated. Figures 5 and 6 give the chordwise vari-

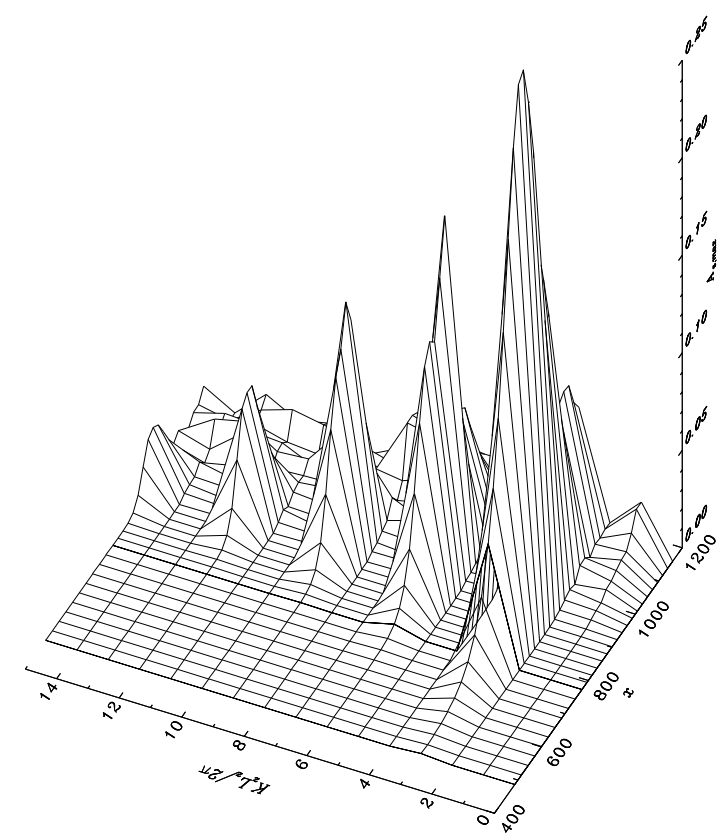

Figure 5. Chordwise development of the spanwise wavenumber spectrum for the LOW-T case.

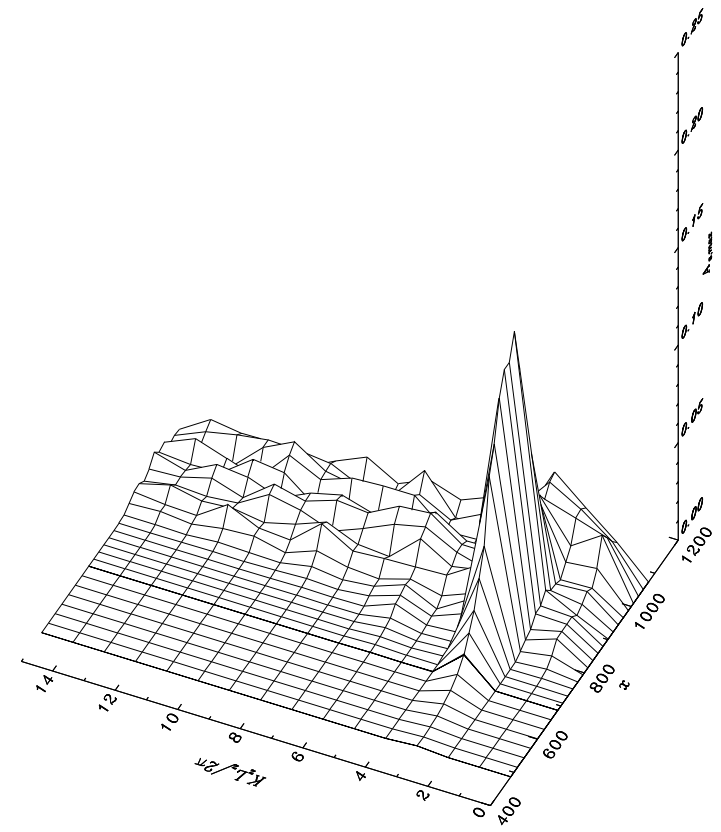

Figure 6. Chordwise development of the spanwise wavenumber spectrum for the HIGH-T case. 
ation of maximum magnitude for each mode defined as

$$
\begin{array}{r}
A_{\max }\left(x, k_{z}\right)=\quad \max \left[A\left(x, y, k_{z}\right)\right], \\
y \in\left[0, y_{\max }\right]
\end{array}
$$

where $A\left(x, y, k_{z}\right)$ is the magnitude of mode $k_{z}$ at point $(x, y)$. The wavenumber of the primary crossflow mode (denoted by $K_{z}$ ) satisfies the relationship $K_{z} L_{z} / 2 \pi=3$, since the steady suction/blowing has three periods over the spanwise box length. In the lower-amplitude traveling-wave case the primary crossflow mode reaches an amplitude equal to $28 \%$ of the freestream velocity prior to transition, while the second harmonic $2 K_{z}$ attains the peak magnitude of $18 \%$. It is also evident in the figure that the appearance of higher harmonics lags behind that of lower ones, and that their magnitudes decrease as the wavenumber increases. This suggests that the growth of higher harmonics is not due to the secondary instability analyzed by Reed (1987), but to the nonlinear wave interactions between the stationary vortices themselves (the second harmonic is excited by the interaction between the primary wave and itself, the third harmonic by the the primary mode and the second harmonic, etc.) The saturation of the stationary vortices must also be caused by these nonlinear wave interactions, since the amplitude of the traveling waves is still small at this stage. In fact, Huai (1996) found a higher growth rate of secondary instability for the traveling modes, since the stationary vortices have reached finite amplitudes. In the higher-amplitude travelingmode case (Fig. 6), however, the higher harmonics never amplify to the same magnitude, and the primary wave itself only reaches about $14 \%$ of the freestream velocity, due to early nonlinear wave interactions of stationary/traveling type.

The chordwise growth of several harmonics and of the spanwise-averaged perturbation velocity, the zeroth wavenumber mode, are shown in Fig. 7. In the LOW-T case (Fig. 7a) the fundamental mode grows exponentially $\left(\alpha_{i}=11\right)$ and is the largest one because the steady suction and blowing generates only this single mode; the second harmonic, $2 K_{z}$, has a higher amplification rate $\left(\alpha_{i}=24\right)$, the third harmonic, $3 K_{z}$, generated by the fundamental interacting with the first harmonic mode, has an even higher amplification rate $\left(\alpha_{i}=39\right)$. At $x_{c}=0.39$, the fundamental mode starts to saturate and reaches a peak amplitude $28 \%$ at $x_{c}=0.41$; the second harmonic saturates at approximately the same location,
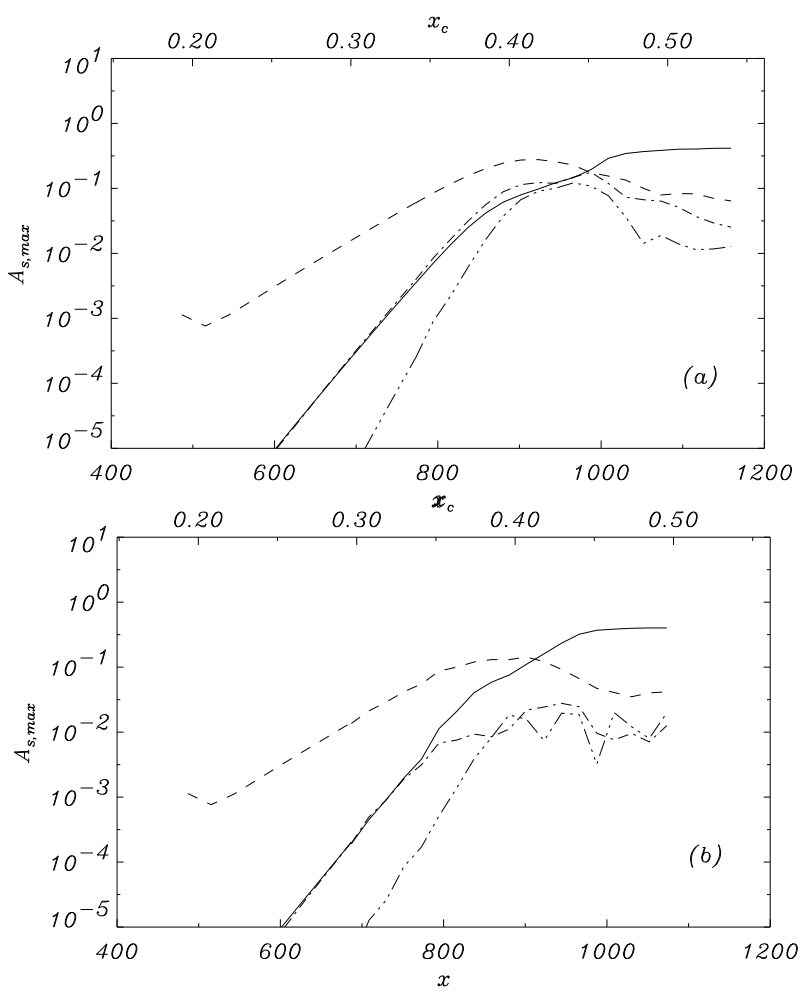

Figure 7. Chordwise development of several stationary modes, - : mean velocity; -- - : $K_{z}$ mode; - - : $2 K_{z}$ mode; $-\cdots-$ : $3 K_{z}$ mode. (a): LOW-T case; (b): HIGH-T case.

while the saturation of the third harmonic occurs downstream of the first- and second-mode saturation point. The mean velocity mode also shows the tendency to saturation when the primary mode saturates, but it regains exponential growth when the transition zone is entered. At the transition location $x_{c}=0.45$, all the modes have amplitudes of the same order of magnitude. In the HIGH-T case (Fig. 7b) the primary mode and the second harmonic have the same initial growth rates as in the LOW-T case, and begin to saturate at $x_{c}=0.36$, while the exponential growth of the third harmonic is sustained further downstream to $x_{c}=0.39$. The higher harmonics are one order of magnitude smaller than the primary mode at saturation, and they are not evident in Fig. 6. The growth of the zeroth wavenumber follows that of the second harmonic very well up to the saturation in both cases.

Figure 8 shows the streamwise velocity contours in the $y z$-planes parallel to the leading edge of the swept wing. At $x_{c}=0.3$, the crossflow vortices are still very weak and the mean flow is uniform in the 


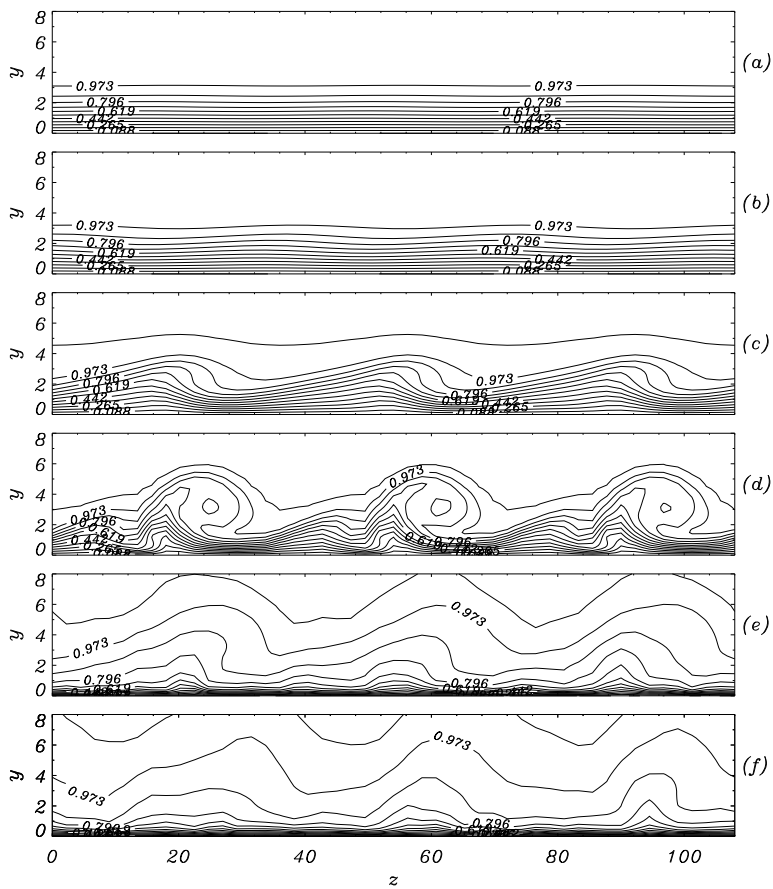

Figure 8. Contours of the time-averaged streamwise velocity $U_{s}$ for the LOW-T case. (a) $x_{c}=0.3$; (b) $x_{c}=0.35 ;$ (c) $x_{c}=0.4 ;$ (d) $x_{c}=0.45 ;$ (e) $x_{c}=0.5$; (f) $x_{c}=0.54$.

spanwise direction. The presence of the crossflow vortices becomes detectable near $x_{c}=0.35$, and the low and high wall-shear regions become very clear at $x_{c}=0.40$. When the transition location $x_{c}=0.45$ is reached (Fig. 8d), the distortion of the mean velocity is so strong that the so-called "half mushroom" structures are observed (Dagenhart, 1992; Joslin and Streett, 1994). This structure (or vortex roll-over state) is accompanied by double inflection points in the wall-normal profiles of the streamwise velocity. Besides the primary alternating wall-shear pattern, weak secondary crossflow vortices can also be observed relatively close to the wall at $z \approx 10,45$ and 80. Malik et al. (1994) also observed these secondary vortices in their numerical study. As the flow goes into the transition and turbulent regions, the strength of the vortical structures gradually diminishes, but the spanwise periodicity remains observable; this explains why the streaks extend into the downstream region in the wall-shear plot (Fig. 1).

For the higher-amplitude traveling-wave case (HIGH-T) the streamwise velocity contours (Fig. 9) show the spanwise distortion of the mean flow, but the "half mushroom" structure that was observed
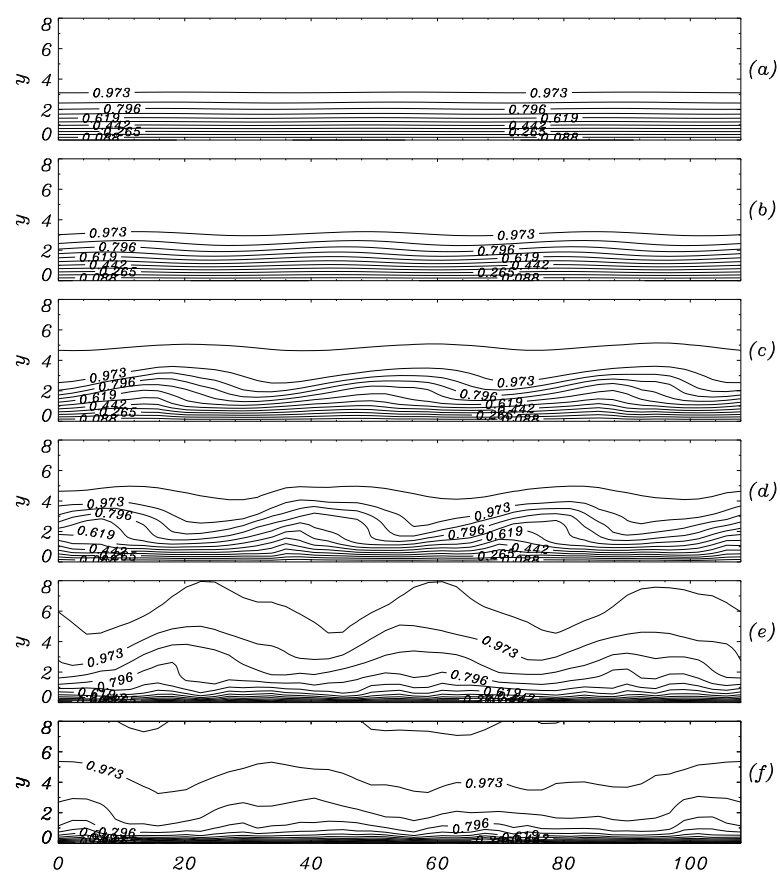

Figure 9. Contours of the time-averaged streamwise velocity $U_{s}$ for the HIGH-T case. (a) $x_{c}=0.3$ (b) $x_{c}=0.35 ;$ (c) $x_{c}=0.4 ;$ (d) $x_{c}=0.42 ;$ (e) $x_{c}=0.45$; (f) $x_{c}=0.5$.

in the LOW-T case is not present because the stationary vortices are not as strong. Correspondingly, the wall-normal velocity profiles in the HIGH-T case exhibit only marginal inflection points.

\section{Frequency analysis}

It has been shown by linear stability theory and experiments (Mack, 1984; Müller and Bippes, 1988; Dagenhart, 1992) that the crossflow velocity profiles present in three-dimensional swept-wing flows can excite both stationary and traveling vortex disturbances, and that the traveling waves have higher growth rates than the stationary ones. In low-turbulence environments such as those encountered in flight conditions, however, receptivity theory (Crouch, 1993) indicates that the stationary disturbances will have much larger amplitudes compared with the traveling modes because of the low free-stream turbulence levels. However, Müller and Bippes (1988) note that the traveling waves play a more important role than the stationary waves in determining the onset of transition. Although the frequency range of the most amplified primary traveling waves can be predicted satisfactorily by the linear stability theory, Kohama et al. (1991) 


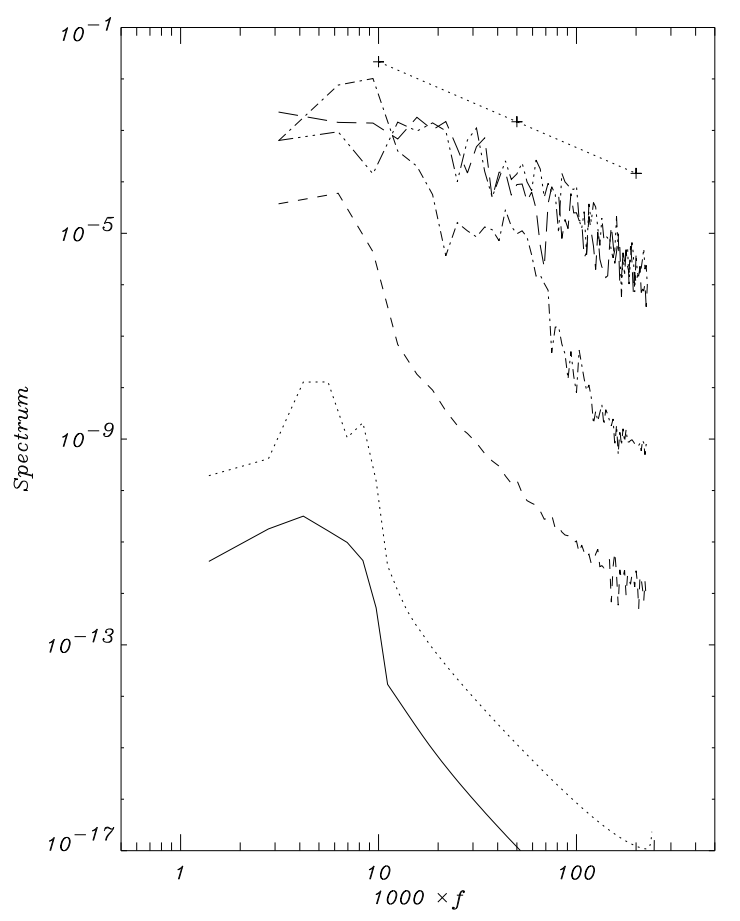

Figure 10. Frequency spectrum of the streamwise velocity $u_{s}$ at the maximum traveling wave locations for the LOW-T case. $-x_{c}=0.3, \cdots \cdots x_{c}=0.35$, - - $x_{c}=0.4,--x_{c}=0.45,-\cdots-x_{c}=0.5$, $---x_{c}=0.54,+f^{-5 / 3}$.

were able to detect a most amplified high-frequency band prior to transition in a swept wing boundarylayer experiment in the ASU low-turbulence wind tunnel $(T u=0.02 \%)$ that could not be predicted by the linear stability theory. This was believed to be due to the secondary instability associated with the strongly distorted streamwise velocity profiles. The same phenomena was reported earlier without explanation by Poll (1985) for a swept-cylinder boundary-layer experiment.

This issue is further studied by examining the development of the frequency spectra of the streamwise fluctuation velocity $u_{s}$. For the LOW-T case (Fig. 10), a low frequency band centered at $f=$ $4.17 \times 10^{-3}$ begins to amplify, in agreement with the prediction of linear stability theory. At $x_{c}=0.35$, however, a second peak is observed in the spectrum, corresponding to the amplification of the second harmonic of the primary traveling wave. The traveling disturbance is dominated by the low-frequency band up until $x_{c}=0.4$, but at $x_{c}=0.45$ a highfrequency band with frequencies one order of magnitude higher is strongly amplified, in agreement with

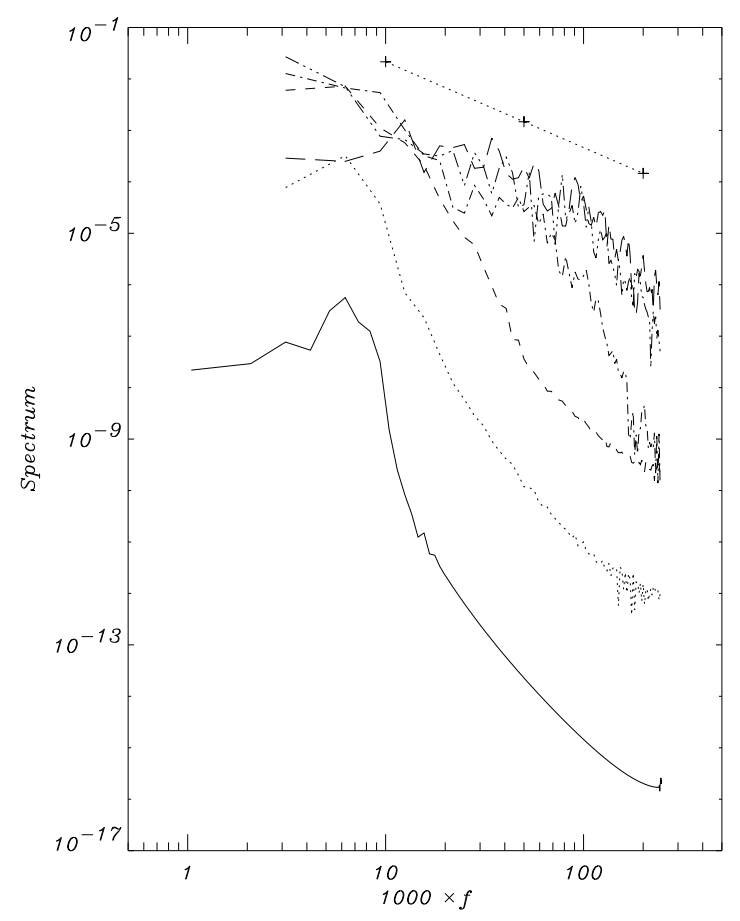

Figure 11. Frequency spectrum of the streamwise velocity $u_{s}$ at the maximum traveling wave locations for the HIGH-T case. $-x_{c}=0.3, \cdots \cdots x_{c}=$ $0.35,---x_{c}=0.4,--x_{c}=0.42,-\cdots-x_{c}=$ $0.45,---x_{c}=0.5,+f^{-5 / 3}$.

the findings of Kohama et al.(1991), and the peak in the spectrum moves to the second harmonic. In the transition and turbulent regions, the distribution of the spectrum becomes typical of a turbulent flow, exhibiting the $-5 / 3$ slope of the inertial subrange. It should be pointed out that due to the grid resolution and accuracy of the numerical scheme, a drop of about four orders of magnitude can be obtained in the spectrum; very small values of the spectrum are produced by either aliasing error or power leakage from higher energy modes and are not physical. However, the LES modeling of the small-scales is producing credible spectral trends.

Figure 11 shows the frequency spectrum for the HIGH-T case. A shift of the peak low-frequency mode occurs at $x_{c}=0.4$. However, the high frequency band is not prominent at the transition location $\left(x_{c}=0.42\right)$; the secondary instability associated with the inflectional points is much weaker because the stationary disturbance is weaker and exhibits only marginal inflection points. Because of the earlier nonlinear stationary/traveling wave interaction the spectrum contents are higher and 
much closer to those of turbulent flows.

\section{Conclusions}

The spatial development of crossflow instability in a $45^{\circ}$ swept-wing boundary layer was studied using the large-eddy simulation (LES) technique. The numerical simulations successfully reproduce the wallshear streaks typical of the crossflow disturbance. The simulations also show that transition occurs later in the simulation with weaker traveling waves, as expected, and that the remnants of the streaks extend deeply into the transition and turbulent regions in the low unsteady-forcing case. The transition locations obtained from the streak patterns compare well with those obtained from the variation of the streamwise skin-friction coefficient. The streaks are approximately aligned in the direction of the external streamline, forming a $4^{\circ}$ differential angle with the freestream, in good agreement with experimental results. Downstream of the transition location the wall shear stress is found to align with the streak direction.

The strength of the stationary vortices is found to grow exponentially, initially independent of the unsteady forcing amplitude; however, it saturates at different levels during the later development, depending on the magnitudes of the traveling vortices. Streamwise velocity contours in the $y z$-planes show the evolution from a wave-like structure to the so-called "half mushroom" structure for the loweramplitude traveling wave (LOW-T) case, leading to double inflection points in the wall-normal velocity profiles prior to transition. However, the "halfmushroom" structure is not observed in the HIGH$\mathrm{T}$ case. The nonlinear interactions involving the stationary vortices are much stronger in the low unsteady-forcing case, as indicated by the amplification of higher harmonics of the primary mode.

The numerical results also show that the traveling waves are dominated early by low frequency components. Prior to transition, in the low unsteadyforcing case a secondary instability band with high frequencies is most amplified, followed by rapid spectrum filling. However, this secondary instability is much weaker in the higher unsteady-forcing case.

\section{Acknowledgments}

This work was sponsored by the NASA Langley Research Center under grant NAG 1-1089. The computer resources were provided by the Numerical Aerodynamic Simulation Facility (NAS).

\section{$\underline{\text { References }}$}

Arnal, D. and Juillen, J. C. (1987) "Three-dimensional transition studies at ONERA/CERT." AIAA Paper 87-1335.

Chorin, A. J. (1968) "Numerical solution of the Navier-Stokes equations." Math. Comput. 22, 745 .

Cooke, J. C. (1950) "The boundary layer of a class of infinite yawed cylinders." Camb. Phil. Soc. 46, 645 .

Crouch, J. D. (1993) "Receptivity of threedimensional boundary layers." AIAA Paper 93-0074.

Dagenhart, J. R. (1992) "Crossflow stability and transition experiments in a swept-wing flow." Ph. D. Dissertation, Virginia Polytechnic Institute and State University.

Germano, M., Piomelli, U., Moin, P., and Cabot, W. H. (1991) "A dynamic subgrid-scale eddy viscosity model." Phys. Fluids A 3, 1760.

Huai, X., (1996) "Large-eddy simulation of laminar-turbulent transition in spatially developing boundary layers." Ph. D. Dissertation, University of Maryland, College Park.

Huai, X., Joslin, R. D., and Piomelli, U. (1994) "Large-eddy simulation of boundary layer transition on swept wings." In Transition, turbulence and combustion, Vol. 1, (M. Y. Hussaini, T. B. Gatski and T. L. Jackson, eds.), 369.

Huai, X., Joslin, R. D., and Piomelli, U. (1997) "Large-eddy simulation of spatial development of transition to turbulence in a two-dimensional boundary layer." Submitted to Theoret. Comput. Fluid Dyn.

Joslin, R. D., Streett, C. L. and Chang, C.-L. (1992) "3-D incompressible spatial direct numerical simulation code validation study - A comparison with linear stability and parabolic stability equation theories for boundary-layer transition on a flat plate." NASA TP-3205. 
Joslin, R. D. and Streett C. L. (1994) "The role of stationary crossflow vortices in boundary-layer transition on swept wings." Phys. Fluids 6, 3442 .

Joslin, R. D. (1995) "Evolution of stationary crossflow vortices in boundary layers on swept wings." AIAA J. 33, 1279.

Kohama, Y., Saric, W. S. and Hoos, J. A. (1991) "A high-frequency secondary instability of crossflow vortices that leads to transition." Proc. R. Aeronaut. Soc. Conf. on BoundaryLayer Transition and Control, Cambridge, UK.

Mack, L. M., (1984) "Boundary-layer linear stability theory," AGARD-R-"709, 3-1.

Malik, M. R., Li, F. and Chang, C. L. (1994) "Crossflow disturbances in three-dimensional boundary layers: nonlinear development, wave interaction and secondary instability." J. Fluid Mech. 268, 1.

Meyer, F. (1987) "Numerische simulation nichtlinearer instabilitäten in einer 3-dimensionalen grenzschicht," DFVLR IB 221-87 A 05.

Müller, B. and Bippes, H. (1988) "Experimental study of instability modes in a threedimensional boundary layer." AGARD-CP438, 13-1.

Nitschke-Kowsky, P. and Bippes, H. (1988) "Instability and transition of a three-dimensional boundary layer on a swept flat plate." Phys. Fluids 31, 786.

Piomelli, U. and Liu, J. (1994) "Large-eddy simulation of rotating channel flows using a localized dynamic model." Phys. Fluids 23, 495.

Poll, D. I. A. (1985) "Some observations of the transition process on the windward face of a long yawed cylinder." J. Fluid Mech. 150, 329.

Pruett, C. D., Zang, T. A., Chang, C. L. and Carpenter, M. H. (1995) "Spatial direct numerical simulation of high-speed boundary-layer flows - part I: algorithmic considerations and validation." Theoret. Comput. Fluid Dyn. 7, 49.

Reed, H. L. (1987) "Wave interactions in sweptwing flows." Phys. Fluids 31, 3419.
Saric, W. S. and Yeates, L. G. (1985) "Generation of crossflow vortices in a three-dimensional flatplate flow." Laminar-Turbulent Transition, (V. V. Kozlov, ed.), (Springer, New York), 429.

Spalart, P. R. (1986) "Direct Simulation of a Turbulent Boundary Layer up to $R e_{\theta}=1410$." J./ Fluid Mech. 187, 61.

Streett, C. L., and Macaraeg, M. G. (1989) "Spectral multi-domain for large-scale fluid dynamics simulations." Int. J. Applied Num. Math. 6, 123 . 